\title{
Is the Geniculostriate System a Prerequisite for Nystagmus?
}

\author{
ALISTAIR R. FIELDER AND NICHOLAS M. EVANS \\ Leicester
}

\section{Summary}

The time of onset of congenital horizontal nystagmus is usually known. We present a group of infants in whom this was determined objectively. In two, vertical nystagmus in the neonatal period became horizontally directed, at 4 months in one, and between 7 and 9 months of age in the second. Two infants (one an oculocutaneous albino) had no visual response initially but subsequently developed horizontal nystagmus as the vision improved at 5 and 6 1/2 months: Type III delayed visual maturation. An infant totally blind due to Norrie's disease was examined in the first week of life but did not develop nystagmus for a further 3 months. Two children with cortical visual impairment never developed nystagmus.

In early infancy, vision is thought to be predominantly subcortical. Therefore the development of nystagmus at a time when the geniculostriate system is emerging functionally (around 3 months), and its absence in cortical visual impairment, has led us to propose a hypothesis suggesting that a functioning geniculostriate system is a prerequisite for the development of horizontal nystagmus.

Even today, nystagmus remains a challenge to both clinician and research worker. Current knowledge of the neurological substrates of eye movements has made it possible to understand more clearly the mechanisms underlying nystagmus associated with certain neurological disorders, but congenital nystagmus $(\mathrm{CN})$, remains largely unexplained. Laboratory scientists, using electronic recording techniques, have demonstrated features inaccessible to the clinician, such as the 12 distinct waveform patterns of $\mathrm{CN}{ }^{1}$ Furthermore they have shown that older classifications into motor and sensory, or pendular and jerk types, have little nosological or clinical value.

The rate of nystagmus reflects the level of vision, albeit only very approximately; slow roving eye movements indicating extremely poor vision, and faster uniplanar horizontal nystagmus a less severe visual deficit. ${ }^{2}$ Both horizontal and roving nystagmus therefore lie along the same spectrum; are not separate entities, and in this article are treated as a single condition. This ensures distinction from the vertical movements seen in 2 of our cases.

To date, the natural history of $\mathrm{CN}$ has been largely neglected, particularly its time of onset. This is due partly to the difficulty in determining this aspect from a parent's history, but also because nystagmus is usually firmly established by the time of the initial ophthalmic examination. Surprisingly, whilst nystagmus is a dramatic sign to the clinician, it may not be differentiated from normal neonatal eye movements and may even pass unnoticed by parents for months. An exception is the presence of a positive family history when a parent is specifically looking for 
this sign $\left({ }^{3,4,5}\right.$,and information obtained by a parent's history, ARF). Consequently, except for this relatively rare instance, we consider that determining the onset of nystagmus by historical means alone is unreliable. The logistics of mounting a prospective study to investigate such an uncommon condition are obvious.

Sustained nystagmus is not seen in children with cortical visual impairment (CVI), and we propose a hypothesis suggesting that a functioning geniculostriate system is a prerequisite for the development of horizontal nystagmus. As will be discussed later, the geniculostriate system is probably not fully functional until about the third month of life, and we will postulate that this determines the time of onset of horizontal nystagmus. It is important to stress that we will focus entirely on the development of constant uniplanar horizontal nystagmus (spontaneous and pathological), so-called 'congenital' nystagmus and including that due to blindness. Physiological nystagmus induced by rotation will not be discussed and we will not consider the transient and ill-sustained nystagmus commonly encountered in neonates, both fullterm and preterm, ${ }^{6,7}$ or that associated with specific neurological disorders. Clinical cases will be used to illustrate the mode of onset of nystagmus and its absence in CVI. In these cases its development, if it occurred, was observed by us.

\section{Patients and Methods}

A complete ophthalmological assessment which included mydriatic refraction was performed on all infants before the onset of nystagmus. For brevity, only positive ocular findings will be mentioned. The vision of infants and young children was assessed prior to November 1984 using an optokinetic (OKN) tape, but after this time with the acuity card procedure (ACP), an adaptation of the preferential looking technique. ${ }^{8}$ Unless otherwise specified, visual acuities were recorded binocularly. Electrodiagnostic tests, electroretinogram (ERG) and visually evoked potential (VEP) to a flash stimulus, were performed without sedation and using skin electrodes. ${ }^{9}$

\section{Case Histories}

Horizontal nystagmus presenting initially as a vertical oscillation

Case 1. A male caucasian infant born at term without complications; he presented at 3 months of age with absence of visual interest, coarse vertical nystagmus and a left convergent strabismus, but no other abnormal ocular signs. At $4 \frac{1}{2}$ months vision improved and the nystagmus had resolved, but despite this $\mathrm{OKN}$ responses remained very poor. By between 7 and 9 months, nystagmus which was horizontal in all positions of gaze had developed and persists at the age of 5 years. He is myopic (right -1.50/-3.0@90, left -1.50/-2.50@90), and corrected visual acuities (Snellen) are: binocular $6 / 12$, right $6 / 18$, left $6 / 24$. At presentation the ERG was normal but the latency of the VEP was delayed.

Case 2. This fullterm caucasian male developed fits in the neonatal period which required treatment with phenobarbitone for a few days. Mother and a friend noted vertical nystagmus within 24 hours of birth. At presentation at 3 months his vision was $1.5 \mathrm{c} . / \mathrm{deg}$. and there were no abnormal ocular findings apart from coarse upbeat nystagmus in all positions of gaze. By 4 months the nystagmus had become horizontally directed and remains unchanged at 6 months of age when acuity is $2.4 \mathrm{c} . / \mathrm{deg}$. Both ERG and VEP were normal.

Comment. These 2 patients who presented in early infancy with coarse vertical nystagmus subsequently developed uniplanar horizontal nystagmus at between 7 and 9 months, and at 4 months respectively.

Delayed visual maturation, concurrent development of nystagmus and visual improvement

Case 3. This Asian male with oculocutaneous albinism was born at term, the product of a consanguineous marriage. When first seen at 8 weeks of age no visual response to any stimulus, including ACP, could be elicited. Nystagmus was not present. At 11 weeks horizontal nystagmus had just developed, and although he was still visually unresponsive vision improved to $3 \mathrm{c} . / \mathrm{deg}$. by the age of 5 months. The nystagmus, horizontal in all positions of gaze, persists. ERG and VEP were not performed. A male cousin born 2 years previously, also an oculocutaneous albino and first examined on the second day of life, behaved similarly, but has since left this area.

Case 4. A female caucasian infant born at 35 weeks gestation by section because of fetal distress, suffered transient neonatal hypoglycaemia 
and jaundice. She presented at $41 / 2$ months visually unresponsive, including an absent response to ACP. Her eyes were divergent and there was no nystagmus or other abnormal ocular finding. At $61 / 2$ months a few beats of horizontal nystagmus were noted but she remained visually unresponsive. Over the next few weeks vision improved to $0.8 \mathrm{c} . / \mathrm{deg}$. and reached $3.0 \mathrm{c} . / \mathrm{deg}$. at 7 months of age, during which time horizontal nystagmus developed with reverse phase OKN horizontally, but normal vertical responses. Just before the improvement of vision the ERG was normal and the VEP exhibited a slightly delayed latency.

Comment. Cases 3 and 4 both had no visual response in early infancy but subsequently improved and are examples of type III delayed visual maturation (DVM). Coincident with or even just preceding this visual improvement, horizontal nystagmus developed and persists.

\section{Nystagmus associated with blindness}

Case 5. This male Asian infant was born at term. There was no consanguinity, but two maternal uncles had been blind from birth, one of whom was mentally retarded. This infant was first seen at 4 days of age at which time bilateral retrolental fibrovascular membranes were noted which precluded fundal examination. Horizontal corneal diameters were: right $10.0 \mathrm{~mm}$., left $9.0 \mathrm{~mm}$., and both anterior chambers were shallow. B-scan ultrasound showed funnel-shaped retinal detachments, with solidification within the triangle. No ERG response could be elicited. A diagnosis of Norrie's disease was made and mother was found to have the characteristic DNA restriction fragment length polymorphism. L1.28. At 8 weeks there was no nystagmus, but this had just developed by 15 weeks and was of a roving type. He has mild developmental delay considered possibly to be secondary to the severe visual handicap.

Comment. Nystagmus developed just after 3 months of age in an infant congenitally, completely and permanently blind.

\section{Cortical visual impairment without nystagmus}

Case 6. This fullterm female caucasian infant had no significant neonatal problems, but within a few weeks she was investigated for poor feeding, failure to thrive, hypotonia and absence of eye-toeye contact. At this time no visual response could be elicited, even to ACP, the eyes were divergent but there were no other abnormal ocular findings and pupillary responses were normal. The ERG was normal but the VEP was delayed and exhi- bited persistence of the simple neonatal waveform. The CT scan showed cortical atrophy. This girl, now 3 years old, has severe developmental delay and major fits followed by infantile spasms all of unknown aetiology. No visual response has ever been elicited, and in the presence of normal pupillary reactions, a normal ERG, and otherwise normal ocular findings, a diagnosis of cortical blindness has been made. Nystagmus has not developed.

Case 7. This $4 \frac{1}{2}$ year old caucasian male child has microcephaly, severe mental retardation and spastic quadriplegia resulting from fetal distress and neonatal birth asphyxia following an uneventful pregnancy. His first ophthalmic examination at 8 months failed to elicit any visual response. Pupil responses were normal, there was a left convergent strabismus but otherwise examination was normal. No ERG could be performed, the VEP was well-formed but showed delay and persistence of the neonatal waveform. A diagnosis of cortical blindness was made. At the age of two years his mother noted some visual improvement and an ACP response of $0.2 \mathrm{c} . / \mathrm{deg}$. was recorded which later improved to $0.8 \mathrm{c}$./deg. Nystagmus has never been observed.

Comment. These two children with severe neurological damage and cortical visual impairment have never developed nystagmus, although in one vision has improved slightly.

\section{Discussion}

We have presented five infants with nystagmus and a further two cases of severe cortical visual impairment who did not develop this sign. The cases included were examined first before the onset of horizontal nystagmus so that its onset could be timed accurately. Inevitably the cases are highly selected and representative rather than comprehensive.

The discussion will be divided into three sections: review of the clinical cases; review of visual development in early infancy; and finally, consideration of the factors which may determine the presence and onset of nystagmus in infancy.

To our knowledge, the progression from vertical to horizontal nystagmus has been reported only once before. ${ }^{10}$ These authors presented 9 cases with vertical nystagmus of whom four had Leber's amaurosis and five oculocutaneous albinism. The nystagmus 
later became horizontally directed in three of the patients with Leber's amaurosis between 3 and 9 months of age. In our two patients the direction altered at 4 (case 2) and 7 months (case 1) and no ocular pathology apart from myopia (case 1) was detected. Other similar cases are known to us and this clinical course is probably not as rare as the dearth of literature would suggest.

Recently, delayed visual maturation (DVM) has been classified into 3 types: I, DVM as an isolated anomaly, or associated either with II, mental retardation, or III, ocular disorders/nystagmus. ${ }^{11,12}$ The two cases presented here (cases 3 and 4) are of type III DVM. Initially both infants were blind, and did not develop nystagmus until a few days before the improvement of vision, and both (and also the cousin of case 3 ) now exhibit the features of $\mathrm{CN}$. This clinical course is common in albinism and was probably first reported by Nettleship in $1909,{ }^{13}$ and later by others. ${ }^{12,14,15}$

The association of nystagmus with a severe congenital visual defect is too well known to require detailed discussion here, but the literature contains few actual observations of the time at which nystagmus begins, other than stating that its onset is around 3 to 4 months. ${ }^{16}$ This is not entirely surprising as most infants with a treatable cause such as cataract undergo surgery early on, whilst most other problems such as Leber's amaurosis, present only very rarely before nystagmus has become established. Our patient with Norrie's disease (case 5) was examined and found to be completely blind in the first week of life and yet did not develop nystagmus for another 14 or 15 weeks.

In contrast to anterior visual pathway pathology, childhood cortical visual impairment (CVI) does not produce sustained nystagmus. ${ }^{2,17,18} \mathrm{~A}$ few beats of nystagmus are common, due either to associated ocular or widespread neurological damage. Cases 6 and 7 in this study both had severe CVI, but the latter did not develop nystagmus despite limited visual improvement, indicating that the absence or presence of vision is not a prerequisite for its development.

It is now appropriate to consider briefly some of the developmental changes taking place in the visual system in the first 3 months of life. Myelination is completed in the thalamocortical and the geniculocalcarine pathways by 4 months postnatally and in the optic nerves by 2 years. Dendrite and spine formation in the lateral geniculate nucleus and visual cortex both increase rapidly from birth to a maximum at 6 months of age. The fovea probably does not reach adult morphology until about 4 years of age. These have been the subject of several recent reviews (e.g. ${ }^{19,20}$ ). Results from preferential looking-based techniques show that visual acuity increases from about $1.0 \mathrm{c} . / \mathrm{deg}$. in the first week of life to about $3.0 \mathrm{c} . / \mathrm{deg}$. at 3 months of age. ${ }^{21}$ This is also the period of most dramatic maturational change for the flash VEP; the latency of the major positive peak decreases from $200 \mathrm{msec}$ to about 100 msec. ${ }^{22,23,24}$ The simple neonatal waveform becomes more complex assuming an adult morphology between 3 and 6 months, ${ }^{22} 6$ and 8 weeks, ${ }^{25} 6$ and 12 weeks, ${ }^{24}$ whilst Barnet and co-workers ${ }^{23}$ noted that $85 \%$ VEPs exhibited notching after the major positive peak after 2 months of age. It is interesting to speculate, but it is not known, whether this VEP progression to a more complex waveform indicates the emergence of cortical function. Although pattern-reversal VEP latency dereases with increasing age from birth onwards, spatial and temporal tuning do not vary greatly over the first 2 months of life. ${ }^{26}$ Recently Braddick and co-workers, ${ }^{27}$ using VEP techniques, identified orientationspecific cortical responses from about 6 weeks of age.

Two other aspects are relevant: monocular OKN symmetry and the development of binocularity. In early infancy there is asymmetry of monocular OKN, thus a stimulus moving in a temporal-to-nasal (TN) direction elicits a response earlier than in a nasal-totemporal (NT) direction. Temporal-to-nasal OKN is probably mediated subcortically, whereas for the NT response a functioning cortex is necessary. ${ }^{28,29}$ Using both an infant's looking behaviour to random-dot or line stereograms and the VEP response to random dot stimuli Braddick and Atkinson ${ }^{28}$ assessed the onset of binocularity to be between 2 and 4 months of age. 
Cortical function increases, then, from about 2 to 3 months of life and the implication is that before this time vision is not mediated by the visual cortex. There is substantial evidence of the existence of two visual systems. Firstly, the geniculostriate system whose function is object identification and which depends on information received from the fovea. Secondly, the subcortical, extrageniculostriate pathway (colliculus-pulvinar-parietal pathway) which is dependent largely on the peripheral retina and is concerned with detection of events, their location, and orientational responses, prior to subsequent identification by the geniculostriate system. ${ }^{\text {see }}{ }^{30}$ Bronson in $1974^{31}$ proposed that visual responses elicited in the first one to two months of life were subserved by the subcortical, phylogenetically older second visual system. Hoffman ${ }^{32}$ using VEP recordings reported a shift in the locus of visual preference behaviour between 6 and 10 weeks of age from a subcortical to a cortical site. Maurer and Lewis ${ }^{33}$ suggested that at birth, the X-pathway to the cortex and the Ypathway to the superior colliculus and pretectum are functional, but the Y-pathway to the cortex is not. When the latter comes into action at about two months there are striking behavioural changes. Later, Dubowitz and co-workers ${ }^{34}$ also suggested that neonatal vision is mediated through subcortical pathways as lesions near the thalamus are more likely to affect visual behaviour than lesions of the occipital cortex. Atkinson ${ }^{35}$ considered there was insufficient evidence to support a fully comprehensive theory of infant vision based on the two systems and she proposed a hypothesis in which maturation of pathways between the cortex and colliculus account for behavioural changes commencing at around two months of age and that cortical binocular function does not emerge until about three months of age. Prior to this, certain aspects of vision are subserved subcortically using a system involving the superior colliculus and other structures, and as stated by Braddick and co-workers ${ }^{27}$ different aspects of cortical function mature at different rates. The relationship between subcortical and cortical (geniculostriate) pathways is probably flexible and likely to be influenced by visual path- way pathology.

The stimulus for the present study was an attempt to find an explanation for the late onset of nystagmus in certain clinical conditions such as DVM type III (e.g. albinism case 3 and congenital nystagmus case 4), following congenital sensory deprivation (Norrie's disease case 5), the alteration of nystagmus direction from vertical to horizontal (cases 1 and 2), and the absence of nystagmus in both type I DVM and CVI (cases 6 and 7). Although there are familial cases of $\mathrm{CN}$ with compelling evidence of the presence of nystagmus from the first week of life, ${ }^{3,4,5}$ there is no recent report of this. Furthermore there have been no documented observations on the direction of nystagmus in the neonatal period: whether horizontal or vertical. In general therefore we concur with $\mathrm{Wybar}^{36}$ and Kommerell and Mehdorn ${ }^{37}$ that horizontal nystagmus is rarely present at birth but becomes manifest after some time (also supported by familial cases in which a parent has specifically looked for this sign).

ACP has permitted the kinetics of DVM to be recorded (see figure in 38). In this condition no evidence of a visual response can be obtained, historically, using OKN or ACP in infancy. Thus these infants are more blind than would be expected from simply a postnatal visual developmental lag (Fielder and Tresidder, personal observation), as even the preterm infant at 35 weeks gestation is capable of responding to a low spatial frequency target grating. Furthermore in type I DVM (DVM as an isolated defect) the visual improvement occurs suddenly, between nine and 19 weeks, or occasionally later if there are significant neonatal problems. ${ }^{12}$ It has been suggested recently that DVM may represent a disorder of subcortical function, ${ }^{12}$ and improvement could therefore signify the emergence of cortical function.

In the title of this paper we pose the question - is the geniculostriate system a prerequisite for nystagmus? As it stands, this is clearly incorrect since in the neonatal period and early infancy this system has still to develop functionally and yet nystagmus occurs (6,7 and our cases 1 and 2). However if the word horizontal (as defined above) is added to the question, the answer we suggest 
becomes affirmative. Neonatal vision is probably largely subcortical and the geniculostriate system does not emerge functionally until about 3 months of age. Visual pathway maturation may be differentially affected according to the type and severity of visual pathway and systemic pathology. In our patients the onset of nystagmus ranged from 3 to 7 months. This would also explain the absence of nystagmus in CVI in which condition the geniculostriate system is damaged and also the absence of this sign in type I DVM which probably represents a defect of the subcortical extrageniculostriate system. We suggest also that the onset of cortical function modifies early onset vertical nystagmus such that it becomes horizontal. The occurrence of nystagmus in neonates, even preterm, obviates the query that the onset of nystagmus after 3 months could be governed by maturation of ocular motor control mechanisms rather than the development of the geniculostriate system.

We have postulated the necessity of a functioning geniculostriate system (but not necessarily functioning normally) for the development of nystagmus. It is beyond the scope of this article to consider the pathogenesis of nystagmus other than to make a few comments. In some there are anomalies of the anterior visual pathway such as achromatopsia, aniridia and optic nerve hypoplasia. In the case of albinism both misrouting of the visual pathway and foveal hypoplasia occur, although Collewijn and co-workers ${ }^{39}$ considered the former the likely cause for the nystagmus. The pathogenesis of $\mathrm{CN}$ not associated with a clinically detectable ocular anomaly is unknown; misrouting has been reported $^{40}$ but not substantiated by subsequent investigation. ${ }^{41} \mathrm{~A}$ defect in the optokinetic system, which normally controls ocular drift, has recently been suggested as the basic defect in both $\mathrm{CN}$ and nystagmus of congenital eye disorders. ${ }^{37}$ It is interesting to note that Baloh and associates ${ }^{42}$ also recorded persistence of monocular OKN asymmetry in achromatopsia.

The difficulties of prospectively studying the natural history of nystagmus in early infancy have already been mentioned, and for this reason we have presented illustrative cases in which the onset of nystagmus is known with certainty. We sought an explanation which might explain the onset of horizontal nystagmus between 3 and 7 to 9 months of age, and its absence before this time, as in type I DVM, and later on, as in CVI. Evidence is accumulating to suggest that in early infancy vision is predominantly subcortical. Therefore the development of nystagmus at a time when the geniculostriate system is emerging functionally, and its absence in CVI in which this system is obviously significantly damaged, has led us to propose a hypothesis suggesting that a functioning geniculostriate system is a prerequisite for the development of horizontal nystagmus. Sustained horizontal nystagmus, as defined earlier, is a distinct entity requiring a degree of geniculostriate function which is not necessary for some other types of nystagmus. Further clinical and neurodevelopmental studies are necessary before this hypothesis can be substantiated, as a whole, in part, or alternatively repudiated.

Dr Merrick Moseley PhD gave helpful advice. Support given by the Wellcome Trust is gratefully acknowledged.

\section{References}

${ }^{1}$ Dell'Osso LF: Congenital nystagmus: basic aspects. In Lennerstrand G, Zee DS, Keller EL eds. Functional basis of ocular motility disorders. Oxford: Pergamon Press 1982: 12938.

2 Jan JE, Farrell K, Wong PK, McCormick AQ: Eye and head movements of visually impaired children. Dev Med Child Neurol 1986, 28: 285-93.

${ }^{3}$ Owen DCL: An illustration of hereditary nystagmus. Ophthalmol Rev 1882, 1: 239-42.

${ }^{4}$ Fisher T: Congenital nystagmus in father and son, Br Med J 1902, 2: 694.

${ }^{5}$ Nettleship E: On some cases of hereditary nystagmus. Trans Ophthalmol Soc UK 1911, 31 : 159-202.

${ }^{6}$ Hoyt CS, Mousel DK, Weber AA: Transient supranuclear disturbances of gaze in healthy neonates. Am J Ophthalmol 1980, 89: 708-13.

${ }^{7}$ Dubowitz LMS, Levene MI, Morante A, Palmer P, Dubowitz V: Neurologic signs in neonatal intraventricular hemorrhage: a correlation with real-time ultrasound. J Pediatr 1981, 9: 127-33.

${ }^{8}$ McDonald MA, Dobson V, Sebris SL, Baitch 
L, Varner D, Teller DY: The acuity card procedure: a rapid test of infant acuity. Invest Ophthalmol Vis Sci 1985, 26: 1158-62.

${ }^{9}$ Fielder AR: Simple pediatric ocular electrodiagnosis. In Smith JL ed. Neuro-ophthalmology focus, 1980. New York. Masson Publishing, 1979:217-27.

${ }^{10}$ Hoyt CS and Gelbart SS: Vertical nystagmus in infants with congenital ocular abnormalities. Ophthalmol Pediatr Genet 1984, 4: 155-62.

${ }^{11}$ Uemera Y, Oguchi Y, Katsumi O: Visual developmental delay. Ophthalmol Paediatr Genet 1981, 1: 49-58.

${ }^{12}$ Fielder AR, Russell-Eggitt IR, Dodd KL, Mellor DH: Delayed visual maturation, Trans Ophthalmol Soc UK 1985, 104: 653-61.

${ }^{13}$ Nettleship E: On some hereditary diseases of the eye. Bowman Lecture, Trans Ophthalmol Soc UK 1909, 29: LVIII-CXCVIII.

${ }^{14}$ Flynn JT: Neonatal ophthalmology: evaluation of visual function in the neonate and infant. In Harley RD ed. Pediatric Ophthalmology. 2nd edition. Philadelphia, WB Saunders Co 1983: $1-27$.

${ }^{15}$ Acers TE: Congenital abnormalities of the optic nerve and related forebrain. Philadelphia. Lea and Febiger, 1983: 17.

${ }^{16}$ Gay AJ, Newman NM, Keltner JL, Stroud MH: Eye movement disorders. Saint Louis. CV Mosby Co. 1974: 63.

${ }^{17}$ Hoyt CS, Nickel BL, Billson FA: Ophthalmological examination of the infant. Developmental aspects. Surv Ophthalmol 1982, 26: $177-89$.

18 Whiting S, Jan JE, Wong PKH, Flodmark O, Farrell K, McCormick AQ: Permanent cortical visual impairment in children. Dev Med Child Neurol 1985, 27: 730-9.

${ }^{19}$ Van Hof-van Duin J and Mohn G: Vision in the preterm infant. In Prechtl HFR ed. Continuity of neural functions from prenatal to postnatal life. Clinics in developmental medicine no. 94. Oxford. Spastics International Medical Publications Ltd., Blackwell Scientific Publications Ltd. 1984: 93-114.

${ }^{20}$ Fielder AR: Ocular growth and the normal development of vision: clinical aspects. Doc Ophthalmol Proc Series 1986, 45: 14-26.

${ }^{21}$ Teller DY, McDonald MA, Preston K, Sebris SL, Dobson V: Assessment of visual acuity in infants and children: the acuity card procedure. Devel Med Child Neurol 1986, 28: 77989.

${ }^{22}$ Ellingson RJ: Development of visual evoked responses in human infants recorded by a response averager. Electroencephalogr Clin Neurophysiol 1966, 21: 403.
${ }^{23}$ Barnet AB, Friedman SL, Weiss IP, Ohlrich ES, Shanks B, Lodge A: VEP development in infancy and early childhood. A longitudinal study. Electroencephalogr Clin Neurophysiol 1980, 49: 476-89.

${ }^{24}$ Fielder AR, Harper MW, Higgins JE, Clarke $\mathrm{CM}$, Corrigan D: The reliability of the VEP in infancy. Ophthalmol Paediatr Genet 1983, 3: $73-82$.

${ }^{25}$ Ferriss GS, Davis GD, Dorsen MMcF, Hackett ER: Changes in latency and form of the photically induced average evoked response in human infants. Electroencephalogr Clin Neurophysiol 1967, 22: 305-12.

${ }^{26}$ Porciatti V: Pattern VEPs in very young infants. Doc Ophthalmol 1986, 45: 99-106.

${ }^{27}$ Braddick OJ, Wattam-Bell J, Atkinson J: Orientation-specific cortical responses in early infancy. Nature 1986, 320: 617-9.

${ }^{28}$ Braddick OJ and Atkinson J: Some recent findings on the development of human binocularity: a review. Behav Brain Res 1983, 10: 14150.

${ }^{29}$ Van Hof-van Duin J and Mohn G: Optokinetic and spontaneous nystagmus in children with neurological disorders. Behav Brain Res 1983, 10: $163-76$.

${ }^{30}$ Hoyt CS: Cortical blindness in infancy. In Pediatric Ophthalmology and Strabismus: Transactions of the New Orleans Academy of Ophthalmology. New York, Raven Press 1986: 235-43.

${ }^{31}$ Bronson G: The postnatal growth of visual capacity. Child Dev 1974, 45: 873-90.

${ }^{32}$ Hoffmann RE: Developmental changes in human infant visual-evoked potentials to patterned stimuli recorded at different scalp locations. Child Dev 1978, 49: 110-18.

${ }^{33}$ Maurer D and Lewis TL: A physiological explanation of infants' early visual development. Canad J Psychol 1979, 33: 232-52.

${ }^{34}$ Dubowitz LMS, Mushin J, de Vreis L, Arden GB: Visual function in the newborn infant: is it cortically mediated? Lancet 1986, 1: 113941.

${ }^{35}$ Atkinson J: Human visual development over the first 6 months of life. A review and a hypothesis. Hum Neurobiol 1984, 3: 61-74.

${ }^{36}$ Wybar KC: Diagnostic and therapeutic problems in nystagmus. Br J Ophthalmol 1969, 53: 224-9.

${ }^{37}$ Kommerell $\mathrm{G}$ and Mehdorn E: Is an optokinetic defect the cause of congenital and latent nystagmus? In Lennerstrand G, Zee DS, Keller EL eds. The functional basis of ocular motility disorders. Oxford, Pergamon Press. 1982: 159-67. 
${ }^{38}$ Fielder AR and Moseley MJ: Do we need to measure the vision of children? J R Soc Med. 1988;81:380-3.

${ }^{39}$ Collewijn $\mathrm{H}$, Apkarian $\mathrm{P}$, Spekreijse $\mathrm{H}$ : The oculomotor behaviour of human albinos. Brain 1985, 108: 1-28.

${ }^{40}$ Meienberg O, Hemphill G, Rosenberg M, Hoyt WF: Visually evoked response asymmetries in a family with congenital nystagmus. Possible evidence of abnormal visual projections. Arch
Neurol 1980, 37: 697-8.

${ }^{41}$ Apkarian $\mathrm{P}$ and Spekreijse $\mathrm{H}$ : The VEP and misrouted pathways in human albinism. In Cracco RQ, Wollner IB eds. Frontiers of clinical neuroscience series. Evoked Potentials. New York. Alan Liss, Inc. 1986: 211-6.

42 Baloh RW, Yee RD, Honrubia V: Optokinetic asymmetry in patients with maldeveloped foveas. Brain Res 1980, 186: 211-16. 